\title{
Intradermal Immunization of BALB/C Mice with Heat Killed Klebsiella pneumoniae ATCC BAA1706 Leads to the Production of both IgG1 and IgG2a
}

\author{
Ítalo Sousa Pereira*, Maria Poliana Leite Galantine, Igor Pereira Ribeiro Muniz, Diego \\ Patrick Soares Lopes, Karine Bitencourt Rodrigues, Lorena Santos Leal, Palloma Porto \\ Almeida, Larissa Rodrigues de Oliveira Sousa and Robson Amaro Augusto da Silva
}

Universidade Federal da Bahia, Instituto Multidisciplinar em Saúde, Campus Anísio Teixeira, Bahia, Brasil

*Corresponding author

\author{
A B S T R A C T
}

\begin{tabular}{|l|}
\hline Ke y w or d s \\
Klebsiella \\
pneumoniae, \\
Production, Heat. \\
\hline Article Info \\
\hline Accepted: \\
19 October 2017 \\
Available Online: \\
10 December 2017 \\
\hline \hline
\end{tabular}

Klebsiella pneumoniae is a gram-negative bacillus commonly isolated from nosocomial pneumonia patients. Given the increasing development of strategies to bypass the therapeutic molecules used today, the development of an immunoprophylactic safe approach against the pathogen is of great clinical relevance. Here we employed the strain of Klebsiella pneumoniae ATCC BAA1706 for the intradermal Immunization of Balb/c mice. Two weeks after three immunizations the mice were intratracheally challenged with $3 \times 10^{8}$ or $1,5 \times 10^{6}$ CFUs of $K$. pneumoniae and euthanized $24 \mathrm{~h}$ post-infection. We did not detect changes in the peripheral blood cell numbers. However, the infection with $3 \times 10^{8}$ CFUs of $K$. pneumoniae led to an increase in the neutrophil count on the bronchoalveolar lavage. The immunization protocol used in this study led to production of $\mathrm{IgG}$ serum titers of IgG1 and IgG2a subclasses against the bacterial antigens. Our results exhibited that the intradermal immunization with heat killed $K$. pneumoniae ATCC BAA1706 led to the activation of the humoral acquired immunity, which has been previously proved participant in K. pneumoniae infections depuration.

\section{Introduction}

Klebsiella pneumoniae is among the leading pathogens causing nosocomial pneumonia and the treatment of its infection is restricted to a few number of therapeutic approaches (Hirsch and Tam, 2010; Sanchez et al., 2013). First described by Friedlander as an encapsulated bacilli isolated from a case of pneumonia in 1882 (Friedlaender, 1882), Klebsiella pneumoniae is a gram negative bacterial pathogen that has great clinical relevance because of the progressive development of evasion mechanisms to therapeutic strategies available today (Kumarasamy et al., 2010; Sanchez et al., 2013).

In addition to pneumonia, $K$. pneumoniae strains have been isolated from other severe medical conditions, such as sepsis, the formation of pyogenic liver abscess, urinary tract infections, meningitis and endophthalmitis (Ho, Tsai et Liao, 2009; 
Yang et al., 2007; Hui et al., 2007; Dhingra, 2008). Among the molecular structures often described related to the pathogenicity of $K$. pneumoniae, the clinical highlights are: $K$. pneumoniae capsular polysaccharide serotypes K1 and K2 (Pan et al., 2008; Clements et al., 2008); Presence of 8 out of the 12 LPS serotypes described (Whitfield et al., 1992); Production of the siderophores aerobactin, enterobactin, salmochelin and yersiniabactin (Hsieh et al., 2008). Production of adhesins such as type 1 and type 3 fimbrae, contributing in biofilm formation processes (Wu et al., 2012).

Intensive care units patients on mechanical ventilation have high susceptibility to pulmonary infections caused by $K$. pneumoniae (Sekowska et al., 2014). Outbreaks of nosocomial $K$. pneumoniae infections occur both in developed countries and developing countries and are considered to be a global public health problem (MunozPrice et al., 2013; Tzouvelekis et al., 2012; Gürntke et al., 2014; Guimaraes and Rocco, 2006; Sekowska, Gospodarek and Kusza, 2014).

The selection of a hyper virulent multidrugresistant strain whose infection has hepatic tropism in Asia in the 1980s and the recent identification of cases of infection with this new strain in North America, South America, Australia, the Caribbean and Africa emphasizes the importance of developing strategies to combat $K$. pneumoniae infections in a non-antibiotics dependent approach (Shon etal., 2013).

The dermal layers are easy access biological sites with great immunological relevance for the abundant presence of Langerhans cells, dendritic cells and other antigen presenting cells (APCs) (Pulit-Penaloza et al., 2014; Levin et al., 2015). During an inflammatory process, these APCs capture and process the invading antigens and then rapidly migrate to secondary lymphoid organs through lymph vessels crossing the dermis. This tissular configuration makes the dermal tissue a good site for the initiation of immune responses (Levin et al., 2015). Immunizations are safe artificial approaches to provide antigens to an organism and it is a known effective way for the selection of vaccines (Wutzler et al., 2013; Kruit et al., 2013).

Many attempts of selecting routes of vaccination with second-generation vaccines against $K$. pneumoniae were recorded in the last decade. However, the use of protein subunits to construct vaccines often requires the incorporation of inflammatory adjuvants for greater effectiveness of the memory (Lee et al., 2015; Lundberg et al., 2013; Kurupati and Poh, 2011; Jain et al., 2015). The use of the whole bacteria for the construction of a vaccine provides a more ample supply of candidates for vaccination (Ahmad et al., 2012), provides a more suitable inflammatory stimulus for the establishment of an immune response and ensures a more complete understanding of the immunological processes related to a natural contact with the pathogen.

In the present study, we performed the intradermal immunization with $K$. pneumoniae ATCC BA1706 inactivated by heat and the subsequent pulmonary infection in BALB / C mice.

\section{Materials and Methods}

Ethical evaluation of the experimental procedure. All animal experimental procedure performed in this study was submitted to and approved by an ethics committee of the Universidade Federal da Bahia, Instituto Multidisciplinar da Saúde Campus Anísio Teixeira, UFBA -IMS CAT. 


\section{Animals}

In this study, we used $24 \mathrm{BALB} / \mathrm{C}$ male mice with 6-8 weeks of age. The animals were obtained from animal facilities of the Universidade Federal da Bahia, Instituto Multidisciplinar da Saúde Campus Anísio Teixeira, UFBA -IMS CAT. The animals had food and water ad libitum throughout all the experimental proceedings.

\section{Bacterial strain}

A sample of the bacterial strain $K$. pneumoniae ATCC BAA-1706 used in this study was kindly provided by the Centro de Pesquisas Gonçalo Moniz- Fiocruz and was preserved frozen at $-6^{\circ} \mathrm{C}$ temperature.

\section{Bacterial culture and CFU determination}

The bacterial sample was thawed to perform scattering cultures in BHI agar culture and MacConkey agar culture whenever requested during the experimental procedure.

All bacterial cultures were maintained at $37^{\circ} \mathrm{C}$ and had a total cultivation time of 24 hours before use to ensure that the bacteria used in all experimental steps were at the same growth stage.

The acquisition of the CFU used was made by spectrophotometry. Bacterial colonies were diluted in $1 \mathrm{~mL}$ sterile saline to obtain a bacterial arbitrary concentration. An aliquot not exceeding the volume of $10 \mu \mathrm{L}$ of this solution was solubilized in $990 \mu \mathrm{L}$ of saline into an optical glass cuvette and then this cuvette was conducted to a spectrophotometer to obtain $0,135 \mathrm{~A}(660 \mathrm{~nm})$, indicating the achievement of $1,5 \times 10^{8} \mathrm{CFU}$ of bacteria (McFARLAND, 1907). Additionally, for the immunizations usage, serial dilutions were made in sterile saline to obtain $1.5 \times 10^{6} \mathrm{CFU}$; for the challenge, we conducted a concentration to obtain $3 \times 10^{8}$ UFC in $50 \mu \mathrm{L}$ of sterile saline.

\section{Immunizations}

Three intradermal immunizations were performed in the right ear of the mice with an interval of 14 days between each immunization. K. pneumoniae was cultured for 24 hours prior to each immunization. After obtaining the desired bacterial load, the bacteria were heat inactivated by subjection to a temperature of $60{ }^{\circ} \mathrm{C}$ for 30 minutes. The animals were anesthetized with intraperitoneal inoculum of ketamine and xylazine adjusted to the body weight of mice. The intradermal inoculum in the ear of the mice was done with an insulin syringe BD Ultra-Fine ${ }^{\mathrm{TM}}(31 \mathrm{G})$. Each animal received $10 \mathrm{uL}$ of bacterial solution $\left(1,5 \times 10^{6} \mathrm{CFU} /\right.$ $\mathrm{mL}$ ) or $10 \mu \mathrm{L}$ sterile saline in each of the three immunizations.

\section{Intratracheal challenge and euthanasia}

Two weeks after the last immunization the animals were challenged intratracheally with $3 \times 10^{8} \mathrm{CFU}$ of $K$. pneumoniae or carried to the intratracheal inoculum of sterile saline. The mice were anesthetized with intraperitoneal xylazine and ketamine solution inoculum (ketamine $100 \mathrm{mg} / \mathrm{kg}$; xylazine $12 \mathrm{mg} / \mathrm{kg}$ ). Then we performed the intratracheal inoculum of $50 \mu \mathrm{L}$ of the bacterial solution or sterile saline as adapted from Viscardi (Viscardi et al., 2002). 24 hours after challenge, the animals were euthanized by overdosage of ketamine and xylazine.

\section{Clinical samples}

After euthanasia, blood samples were obtained for total cell count diluted in Turk's solution by light microscopy in Neubauer chamber. Blood samples were also obtained to perform blood smears stained with Panotic 
and to obtain blood serum. The tracheas of mice were exposed and channeled with a catheter attached to a needle. After channeled, $0.5 \mathrm{~mL}$ of sterile saline was inserted and recovered from the lungs of mice three times to obtain bronchoalveolar lavage (BAL), achieving a total of volume of $1.5 \mathrm{~mL}$ of sterile saline entered the lungs. The lungs of the mice were removed, fixed in formaldehyde (10\%) and sent for histopathologic analysis.

The BAL of each mouse had its number of total cells counted by light microscopy in Neubauer chamber after dilution $(1: 10)$ in Turk's solution. BAL cells were concentrated by citospin and stained with Panoptic dye for the differentiation of the cellular profile using morphometric differentiation criteria.

\section{Antibodies ELISA}

Blood sera of the mice were submitted for evaluation of optical density concerning the serum titers of IgG1 and IgG2a antibodies by ELISA. For this evaluation, the plates were coated with bacteria subjected to the same culture conditions of the other experimental procedures.

\section{Statistical analysis}

Our results were evaluated using the nonparametric test Kruskal-Wallis with the multiple comparison posttest of Dunn or the nonparametric test of Mann-Whitney for obtaining the p-value. The comparison was considered significant if the obtained $\mathrm{p}$-value was less than $0.05(<0,05)$. All statistical analyzes were performed with the GraphPad Prism software version 6.0.

\section{Results and Discussion}

Twenty-four hours following the intratracheal infection, the mice were euthanized. The total number of nucleated cells in the peripheral blood of the mice was determined by light microscopy after the dilution of the peripheral blood in Turk's solution. We did not find any difference between the total numbers of nucleated cells in the peripheral blood of mice between the evaluated groups (Figure 1).

In addition, we made blood smears with aliquots of blood. We did not find differences between the evaluated groups concerning to blood leukocytes of the peripheral blood of mice (Figure 2).

The lungs of the mice were cannulated by the trachea and we made pulmonary lavages with sterile saline to obtain the BAL.

The total number of cells in the BAL was obtained by Neubauer chamber counting after dilution in Turk solution. We observed differences only between the group infected with $3 \times 10^{8}$ CFU of bacteria and the uninfected group (Figure 3). Indicating an increase in the number of cells in the lung parenchyma $24 \mathrm{~h}$ after infection with a high dose $\left(3 \times 10^{8} \mathrm{CFU}\right)$ of $K$. pneumoniae.

Infection with the higher dose of $K$. pneumoniae has led to the increased number of neutrophils in the BAL cells regardless of immunization (Figure 4).

The serum from the peripheral blood of the mice was obtained and conducted to the dosage of the relative titers of the antibodies IgG1 and IgG2a by ELISA. Intradermal immunization led to the production of titers of anti-K. pneumoniae antibody present in the sera of mice (Figure 5a and 5b).

As found by others infections protocols with $K$. pneumoniae, our results didn't report any differences regarding the total number of nucleated cells in the peripheral blood (Figure 1) (Woods et al., 2007). 
Interestingly, our infection protocol did not lead to differences concerning to blood leukocytes in the mice peripheral blood. This blood leukocytes pattern isn't commonly seen in the peripheral blood of mice infected by others strains of $K$. pneumoniae (Figure 2) (Gardner et al., 2014; Woods et al., 2007).

The absence of changes in total counts and differentials identified in this study, even when the mice were infected with high microorganism doses $\left(3 \times 10^{8}\right.$ UFC), may signal that infections by ATCC BAA-1706 strain does not cause systemic leukocyte changes 24 hours after infection. This might indicate that the bacterial antigenic stimulation caused by the infection may have been stopped independently from immunization $24 \mathrm{~h}$ after infection. This hypothesis is supported by the absence of Klebsiella pneumoniae colonies observed in the culture of lungs macerate in our study (data not shown) and by the absence of difference in total number of neutrophils observed in our data (Figure 2). These phagocytes are remarkable in infectious processes that involve extracellular microorganisms such as Klebsiella pneumoniae (Xiong et al., 2015; McIntyre et al., 1989; Villa-Ambriz et al., 2012).

They constitute the first phagocytic leukocytes population to be recruited to the infection site and for the production of extracellular traps (NETs). NETs are neutrophil derived structures consisting of DNA fibrils conjugated to contents of the neutrophil granules, such as broad spectrum proteases that can kill pathogens even without phagocytosis activity (Brinkmann et al., 2004). Such mechanism has already proven itself participant in $K$. pneumoniae and other respiratory pathogens infections (Sharma et al., 2014; Yoo et al., 2014).

Fig.1 Total number of nucleated peripheral blood cells. BALB / c mice were immunized intradermally with $10^{6} \mathrm{CFU}$ of Klebsiella pneumoniae three times with a two-week interval between each immunization. Two weeks after the last immunization the mice were infected intratracheally with either $3 \times 10^{8}$ or $1.5 \times 10^{6} \mathrm{CFU}$ of $K$. pneumoniae and then they were euthanized $24 \mathrm{~h}$ later. Blood samples were collected and conducted to the total cell counting in a Neubauer chamber. The data were obtained from two independent experiments $(n=3-6$ per group; plot: Mean with SEM)

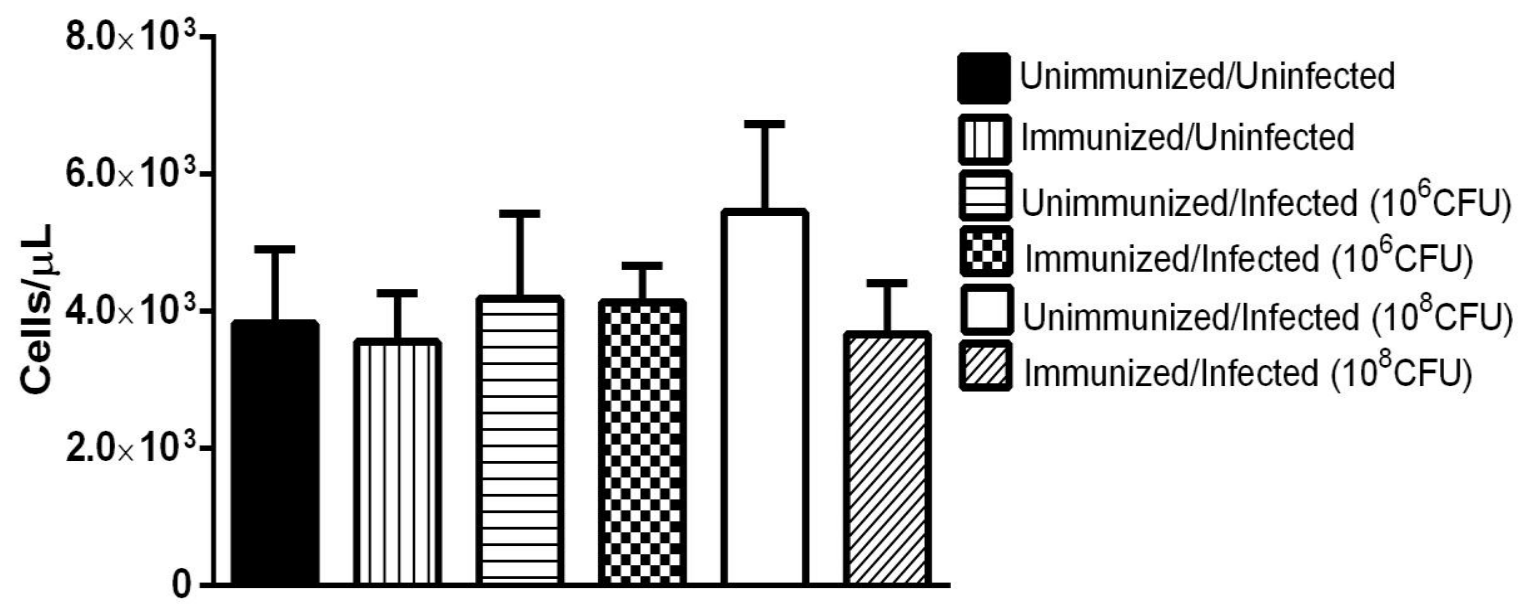


Fig.2 Absolute differential count of peripheral blood leukocytes. BALB / c mice were immunized intradermally with $106 \mathrm{CFU}$ of Klebsiella pneumoniae three times with a two week interval between each immunization. Two weeks after the last immunization the mice were infected intratracheally with $3 \times 10^{8}$ or $1.5 \times 10^{6} \mathrm{CFU}$ of $K$. pneumoniae and they were euthanized $24 \mathrm{~h}$ later. Blood smears were made and differential count following morphometric standards was made by light microscopy. Data were obtained from two independent experiments $(n=3-6$ per group; plot: Mean with SEM)

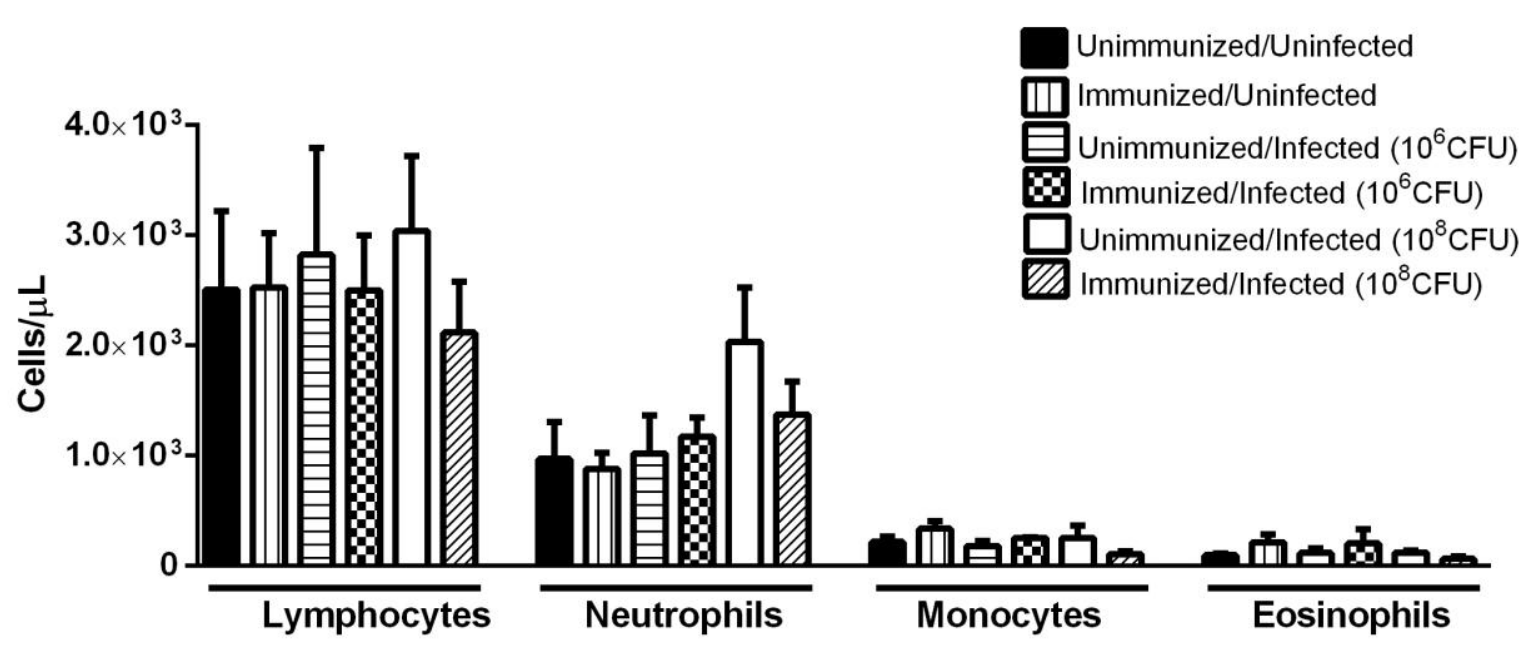

Fig.3 Total count of nucleated cells in the bronchoalveolar lavage. BALB / c mice were immunized intradermally with $10^{6} \mathrm{CFU}$ of Klebsiella pneumoniae three times with a two week interval between each immunization. Two weeks after the last immunization the mice were infected intratracheally with $3 \times 10^{8}$ or $1.5 \times 10^{6} \mathrm{CFU}$ of $K$. pneumoniae and they were euthanized $24 \mathrm{~h}$ later. The lungs of mice were cannulated through the trachea and sterile saline was inserted for obtaining the BAL. The total number of cells was established by Neubauer chamber counting by light microscopy. The unimmunized mice infected with $3 \times 10^{8}$ UFC of $K$. pneumoniae showed greater total cell count than the unimmunized and uninfected group. Data were obtained from two independent experiments ( $n=3-6$ per group, $p=0.0357$; plot: Mean with SEM)

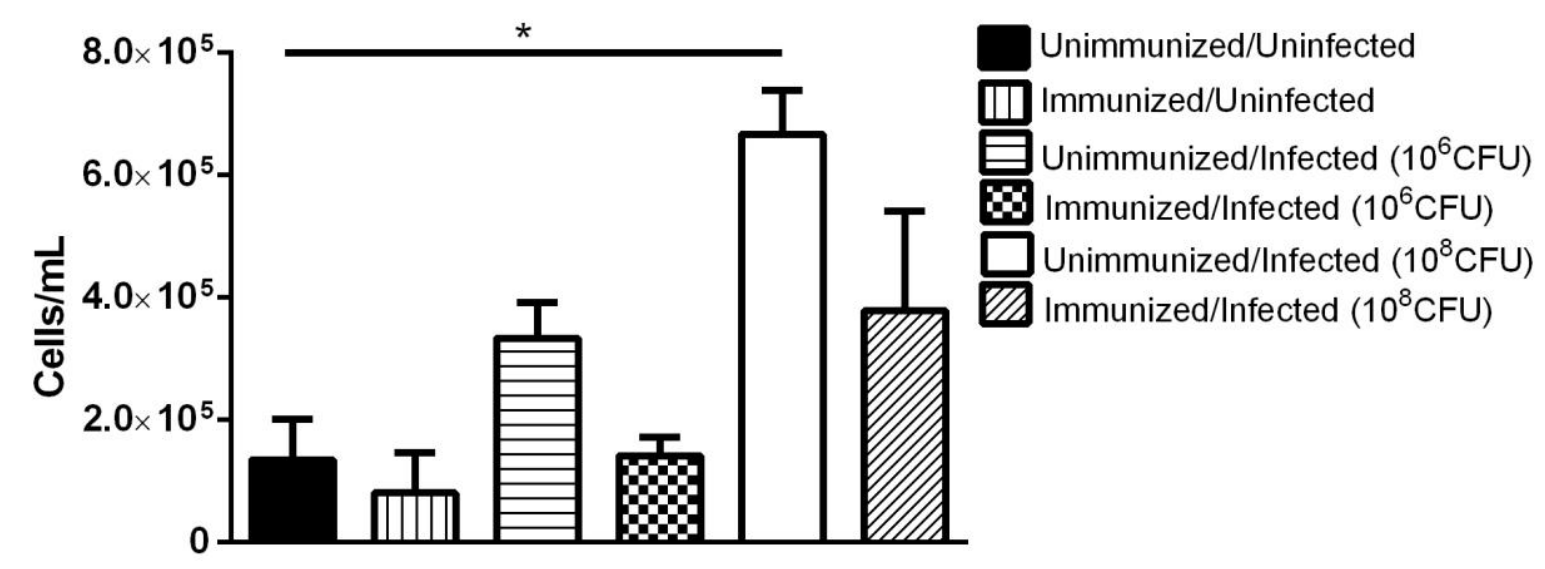


Fig.4 Differential count of nucleated cells in the bronchoalveolar lavage. BALB / C mice were immunized intradermally with $106 \mathrm{CFU}$ of Klebsiella pneumoniae three times with a two week interval between each immunization. Two weeks after the last immunization the mice were infected intratracheally with $3 \times 10^{8}$ or $1.5 \times 10^{6} \mathrm{CFU}$ of $\mathrm{K}$. pneumoniae and they were euthanized $24 \mathrm{~h}$ later. The lungs of mice were cannulated through the trachea and sterile saline was inserted for obtaining the BAL. Cells were stained and counted following morphometric differentiation patterns. Mice infected with $3 \times 10^{8} \mathrm{CFU}$ of $K$. pneumoniae showed higher neutrophil count than the immunized and uninfected group. Data were obtained from two independent experiments (n = 3-6 per group, $\mathrm{p}=0.0043$; plot: Mean with SEM)

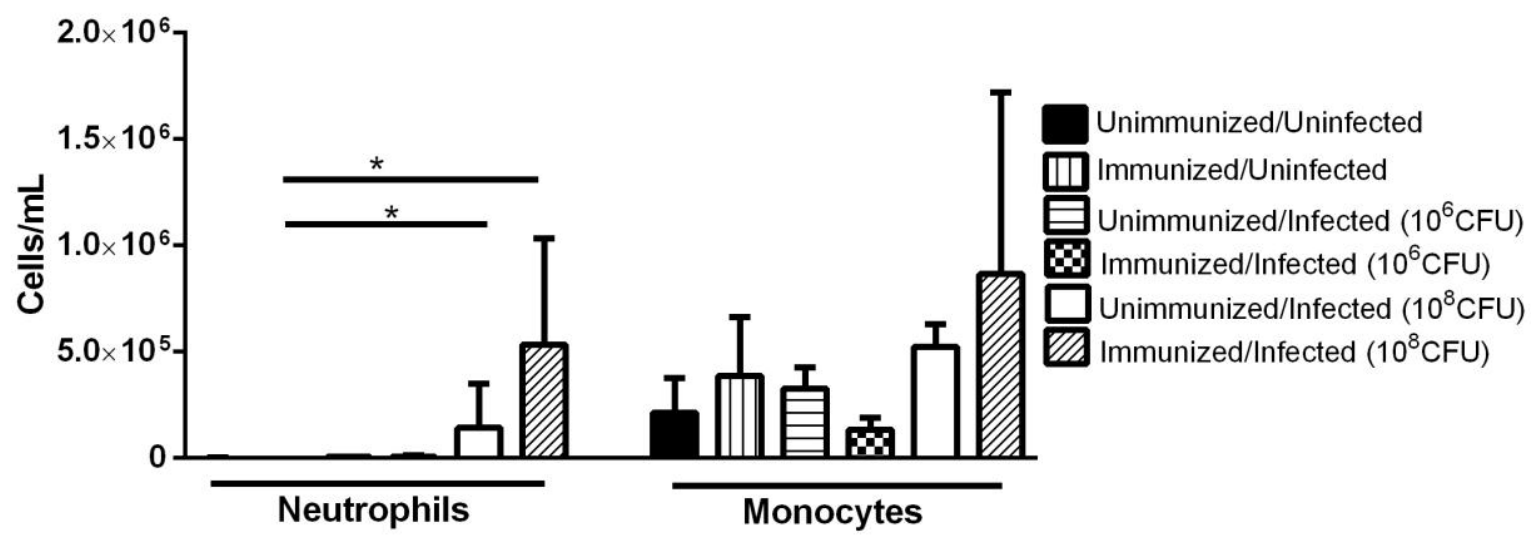

Fig.5 Optical Density from serum titers of anti-K. pneumoniae antibodies. BALB / c mice were immunized intradermally with $10^{6} \mathrm{CFU}$ of Klebsiella pneumoniae three times with a two week interval between each immunization. Two weeks after the last immunization the mice were inoculated intratracheally with sterile saline and they were euthanized $24 \mathrm{~h}$ later. The blood serum was obtained by centrifugation of peripheral blood. A: Optical density of serum titers of IgG1 in mice sera. B: Optical density of serum titers of IgG2a in mice sera. The mice immunized with $K$. pneumoniae showed greater optical density (OD) of serum antibodies than unimmunized mice for both $\operatorname{IgG} 1(\mathrm{p}=0.0022)$, and $\operatorname{IgG} 2 \mathrm{a}(\mathrm{p}=0.0043)$. Data were obtained from two independent experiments $(n=3-6$ per group; plot: Mean with SEM)

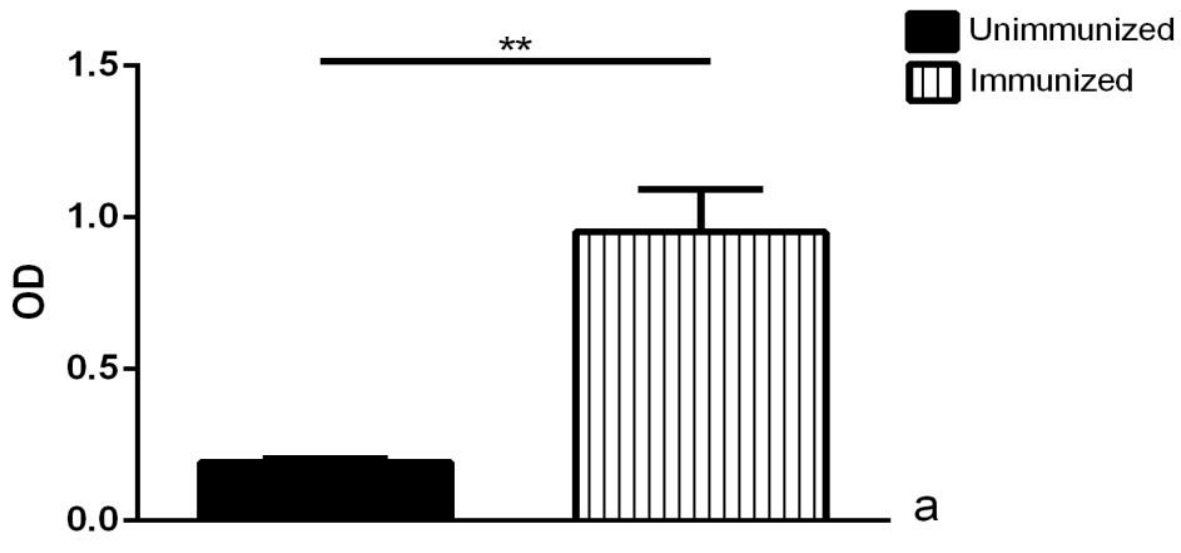




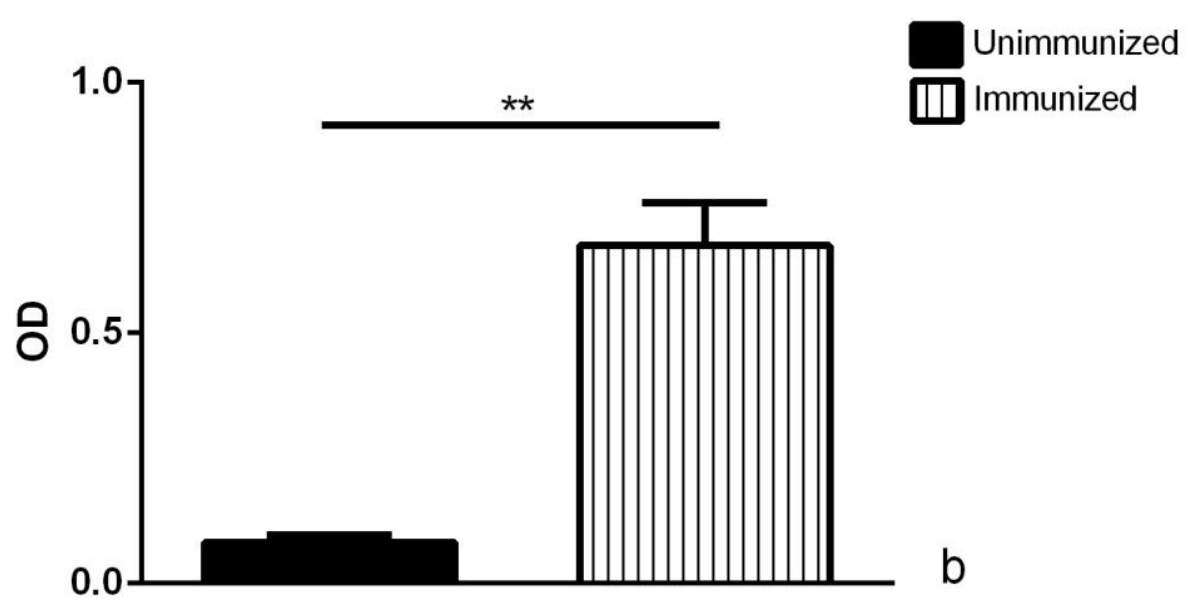

We observed differences only between the group infected with $3 \times 10^{8} \mathrm{CFU}$ of bacteria and the uninfected group (Figure 3). Indicating an increase in the number of cells in the lung parenchyma $24 \mathrm{~h}$ after infection with a high dose $\left(3 \times 10^{8} \mathrm{CFU}\right)$ of $K$. pneumoniae.

The increase in the number of cells of BAL is remarkable in murine $K$. pneumoniae infections (Dolgachev et al., 2012; Soares et al., 2003). However, in our experimental procedure only the dose of $3 \times 10^{8} \mathrm{CFU}$ of $K$. pneumoniae was able to show an increase in the number of BAL cells (Figure 3).

Here, we could observe an increase in the neutrophils numbers in the BAL, regardless of immunization.This increase in polymorphonuclear counts in BAL may reflect the importance of these cells in the clearance of pathogens in infections by extracellular microorganisms (Xiong et al., 2015).

The strain of $K$. pneumoniae used in this study is proven to be deficient of blaKPC genes whose expression is responsible for the production carbapenemases, important proteins for the maintenance of infections. $K$. pneumoniae strains harboring this gene are involved in outbreaks throughout the world (Partridge et al., 2015; Liu et al., 2014). The use of a strain not carrying many antibiotic resistance mechanisms for the development of a first generation vaccine provides increased safety and can prevent the development of some outbreaks of infections worldwide.

Antibodies are protein molecules produced by plasma cells after presentation of an invading antigen. These molecules participate in various effector mechanisms in the clearance of pathogens, such as the activation of $\mathrm{Clq}$ enzyme fragment of the classical pathway of the complement system. Activation of the complement system is a participant in the clearance of pathogens by the pathogen opsonization with $\mathrm{C} 3 \mathrm{~b}$ protein and the formation of C5b6789 (attack complex membrane, MAC) (Nypaver et al., 2010). The activation of complement by direct cleavage of C3 by bacterial surface structures, such as LPS and outer membrane proteins, initiates the complement cascade by the alternative pathway. This activation of the complement system alternative pathway is prevalent in early stages of infection, while the activation of the classical and lectin pathway is present for prolonged infections (Nypaver et al., 2010). Previous studies have shown that some strains of $K$. pneumoniae, which possesses 
certain serotypes of LPS (such as K1, K10 and K16), exhibited resistance mechanisms against the recognition and effector functions of the complement system (Merino et al., 1992; Alberti et al., 1996). However, when analyzing the proliferation of the bacteria in the scenario of absence of Factor B, the lack of activation of the classical pathway of the complement system seemed to support the bacterial proliferation almost exponentially, this result as also observed in a C5 depletion setup (Nypaver et al., 2010). Additionally, the intraperitoneal adoptive transference of the sera of mice immunized with extracellular vesicles of $K$. pneumoniae, led to protection from the lethality induced by the intraperitoneal challenge of a high dose of $K$. pneumoniae (Lee et al., 2015). Taken together, these results suggests that even though some strains of $K$. pneumoniae possesses resistant mechanisms against the complement system, it activation plays a major role in $K$. pneumoniae infection control. Our immunization protocol led to the production of serum titers of both, IgG1 (Figure 5a) and IgG2 (Figure 5b). These antibodies in the blood serum can be crucial to the activation of the classical pathway of the complement system in the early stages of infection, thus the efficient depuration of the bacteria.

Given it capacity of recruiting polymorphonucleated leukocytes, It is hypothesized that the ideal host protective defense against Klebsiella pneumoniae infection is based on the production of memory Th17 cells (Disteldorf et al., 2015). We did not assess the differentiation of naïve lymphocytes in Th17 cells. However, other studies have shown that there are ways to achieve protection against $K$. pneumoniae in a non-IL17 dependent manner (Tsao et al., 2015; Lee et al., 2015). The activation of the complement system pathways lead to the fragmentation of $\mathrm{C} 5$, thus the production of the fragment $\mathrm{C} 5 \mathrm{a}$, a potent neutrophil chemoattractant (Mayadas, Cullere et Lowell, 2014).

Besides the activation of the complement system, antibodies can opsonize infectious microorganisms through binding of their paratopes at bacterial surface antigens. Therefore, antibodies can stimulate effector functions on neutrophils, which recognize the antigen-antibody complex via Fc receptors (Mayadas, Cullere et Lowell, 2014).

Our immunization protocol led to the production of Klebsiella pneumoniae recognizing antibodies. Even when not immunized, the intratracheal challenge with the used $K$. pneumoniae strain did not lead to lethality, emphasizing the safeness of the usage of ATCC BAA1706 as whole for immunizations protocols. The use of a first generation vaccine provides the supply of a larger number of candidate antigens for the stimulation of B lymphocytes, stimulating the production of a wider variety of antibodies, possibly allowing greater complement system activation and coverage of the surface antigens by anti-K. pneumoniae antibodies and complement fragments, facilitating the recognition of the pathogen by effector cells.

\section{References}

Ahmad, T. A., Sayed, L, H. E., Haroun, M., Hussein, A. A., and Ashry, E. S. H. E. 2012. Development of Immunization Trials against Klebsiella pneumoniae. Vaccine 30(14): 2411-20.

Alberti, S., Alvares, D., Merino, S., Casado, M. T., Vivanco, F., Tomas, J. M., and Benedí, V. J. 1996. Analysis of Complement C3 Deposition and Degradation on Klebsiella pneumoniae. Analysis of Complement C3 Deposition and Degradation on Klebsiella pneumoniae. Infection and Immunity 64(11): 4726-32.

Brinkmann, V., Reichard, U., Goosmann, C., Fauler, B., Uhlemann, Y., Weiss, D. S., 
Weinrauch, Y., and Zychlinsky, A. 2004. Neutrophil Extracellular Traps Kill Bacteria. Science 303(5663): 1532-35.

Clements, A., Gaboriaud, F., Duval, J. F. L., Farn, J. L., Jenney, A. W., Lithgow, T., Wijburg, O. L. C., Hartland, E. L., and Strugnell, R. A. 2008. The Major Surface-Associated Saccharides of Klebsiella pneumoniae Contribute to Host Cell Association. PloS One 3 (11): e3817.

Dhingra, K. R. 2008. A Case of Complicated Urinary Tract Infection: Klebsiella pneumoniae Emphysematous Cystitis Presenting as Abdominal Pain in the Emergency Department., The western journal of emergency medicine 9:171-7.

Disteldorf, E. M., Krebs, C. F., Paust, H. J., Turner, J. E., Nouailles, G., Tittel, A., Schwesinger, A. M. 2015. CXCL5 Drives Neutrophil Recruitment in TH17-Mediated GN. Journal of the American Society of Nephrology: JASN 26(1): 55-66.

Dolgachev, V. A., Yu, B., Reinke, J. M., Raghavendran, K., and Hemmila, M. R. 2012. Host Susceptibility to Gram-Negative Pneumonia after Lung Contusion. The Journal of Trauma and Acute Care Surgery 72(3): 614-22-3.

Friedlaender, C. 1882. Ueber Die Schizomyceten Bei Der Acuten Fibr Sen. Arch Pathol Anat Physiol Klin Med. 87:319-324.

GardnerJ. C., Noel, J. G., Nikolaidis, N. M., Karns, R., Aronow, B. J., Ogle, C. K., and McCormack, F. C. 2014. G-CSF Drives a Posttraumatic Immune Program That Protects the Host from Infection. Journal of Immunology (Baltimore, Md. : 1950) 192(5): 2405-17

Guimaraes,M. M. Q., and Rocco, R.. 2006. Prevalence of Ventilator-Associated Pneumonia in a University Hospital and Prognosis for the Patients Affected. J Bras Pneumol. 32(4): 339-46

Gürntke, S., Kohler, C., Steinmetz, I., Pfeifer, Y., Eller, C., Gastmeier, P., Schwab, F., and Leistner, R. 2014. Molecular Epidemiology of Extended-Spectrum Beta-Lactamase (ESBL)-Positive Klebsiella pneumoniae from Bloodstream Infections and Risk Factors for Mortality. Journal of Infection and Chemotherapy 20(12):817-19.
Hirsch, E. B., and Tam, V. H. 2010. Detection and Treatment Options for Klebsiella pneumoniae Carbapenemases (KPCs): An Emerging Cause of Multidrug-Resistant Infection. Journal of Antimicrobial Chemotherapy 65 (6): 1119-25.

Ho, M. P., Tsai, K. C., and Liao, C. H. 2009. Fatal Klebsiella pneumoniae Meningitis and Concomitant Disseminated Intravascular Coagulation in a Patient with Diabetes Mellitus. Journal of Emergencies, Trauma and Shock 2(2): 135-36.

Hsieh, P. F., Lin, T. L., Lee, C. Z., Tsai, S. F., and Wang, J. T. 2008. Serum- Induced Iron- Acquisition Systems and TonB Contribute to Virulence in Klebsiella pneumoniae Causing Primary Pyogenic Liver Abscess. The Journal of Infectious Diseases 197(12): 1717-27.

Huij. J.Y.H., Yang, M. K. W., Cho, D. H. Y., Li, A., Loke, T. K. L., Chan, J. C. S., and Woo, P. C. Y. 2007. Pyogenic Liver Abscesses Caused by Klebsiella pneumoniae: US Appearance and Aspiration Findings. Radiology 242(3): 769-76.

Jain, R.R., Mehta, M. R., Bannalikar, A. R., and Menon, M. D. 2015. Alginate Microparticles Loaded with Lipopolysaccharide Subunit Antigen for Mucosal Vaccination against Klebsiella pneumoniae. Biologicals 43(3): 195-201.

Kruit, W. H. J., Suciu, S., Dreno, B., Mortier, L., Robert, C., Sileni, V. C., and Maio, M. 2013. Selection of Immunostimulant AS15 for Active Immunization with MAGE-A3 Protein: Results of a Randomized Phase II Study of the European Organisation for Research and Treatment of Cancer Melanoma Group in Metastatic Melanoma. Journal of Clinical Oncology: Official Journal of the American Society of Clinical Oncology 31(19): 2413-20

Kumarasamy, K. K., Toleman, M. A., Walsh, T. R., Bagaria, J., Butt, F., Balakrishnan, R., Chaudhary, U., Doumith, M., Giske, C. G., and Irfan, S. 2010. Emergence of a New Antibiotic Resistance Mechanism in India, Pakistan, and the UK: A Molecular, Biological, and Epidemiological Study. Lancet Infecti. Dis. 10: 597-602

Kurupati, P., Ramachandran, N. P., and Poh, C. L. 
2011. Protective Efficacy of DNA Vaccines Encoding Outer Membrane Protein A and OmpK36 of Klebsiella pneumoniae in Mice. Clinical and Vaccine Immunology: CVI 18(1): 82-88

Lee, W. H., Choi, H. I., Hong, S. W., Kim, K. S., Gho, Y. S., and Jeon, S. G. 2015. Vaccination with Klebsiella pneumoniae Derived Extracellular Vesicles Protects against Bacteria-Induced Lethality via Both Humoral and Cellular Immunity. Nature 47 (9):183-9

Levin, C., Perrin, H., and Combadiere, B. 2015. Tailored Immunity by Skin AntigenPresenting Cells. Human Vaccines and Immunotherapeutics 11(1): 1-10

Liu, Y., Li, X. Y., Wan, L. G., Jiang, W. Y., Yang, J. H., and Li, F. Q. 2014. Virulence and Transferability of Resistance Determinants in a Novel Klebsiella pneumoniae Sequence Type 1137 in China. Microbial Drug Resistance (Larchmont, N.Y.) 20(2): $150-55$

Lundberg, Urban, Beatrice M Senn, Wolfgang Schüler, Andreas Meinke, and Markus Hanner. 2013. Identification and Characterization of Antigens as Vaccine Candidates against Klebsiella pneumoniae. Human Vaccines \& Immunotherapeutics 9(3): 497-505. doi:10.4161/hv.23225.

Mayadas, T. N., Cullere, X., and Lowell, C. A. 2014. The Multifaceted Functions of Neutrophils. Annual Review of Pathology 9:181-218.

McFarland, J. 1907. The nephelometer: An Instrument For Estimating The Number Of Bacteria In Suspensions Used For Calculating The Opsonic Index And For Vaccines. JAMA: The Journal of the American Medical Association XLIX (14). American Medical Association: 1176. doi:10.1001/jama.1907.253201400 22001f.

McIntyre, K. W., Unowsky, J., DeLorenzo, W., and Benjamin, W. 1989. Enhancement of Antibacterial Resistance of Neutropenic, Bone Marrow-Suppressed Mice by Interleukin-1 Infection and Immunity 57(1): $48-54$.

Merino, S., Camprubi, S., Alberti S., Benedi, V. J., and Tomas, J. M. 1992. Mechanisms of Klebsiella pneumoniae Resistance to
Complement-Mediated Killing. Infection and Immunity 60(6): 2529-35.

Munoz-Price, L. S., Poirel, L., Bonomo, R. A., Schwaber, M. J., Daikos, G. L., Cormican, M., Cornaglia, G. 2013. Clinical Epidemiology of the Global Expansion of Klebsiella pneumoniae Carbapenemases. The Lancet Infectious Diseases 13(9): 78596.

Nypaver, Christina M., Margaret M. Thornton, Suellen M. Yin, David O. Bracho, Patrick W. Nelson, Alan E. Jones, David M. Bortz, and David M. Younger. 2010. Dynamics of Human Complement-Mediated Killing of Klebsiella pneumoniae. American Journal of Respiratory Cell and Molecular Biology 43(5): 585-90.

Pan, Y.J., Fang H. C., Yang H. C., Lin T. L., Hsieh, P. F., Tsai, F. C., Keynan, Y., and Wang, J. T. 2008. Capsular Polysaccharide Synthesis Regions in Klebsiella pneumoniae Serotype K57 and a New Capsular Serotype. Journal of Clinical Microbiology 46(7): 2231-40.

Partridge, S. R., Ginn, A. N., Wiklendt, A. M., Ellem, J., Wong, J. S. J., Ingram, P, Guy, S., Garner, S., and Iredell, J. R. 2015. Emergence of blaKPC Carbapenemase Genes in Australia. International Journal of Antimicrobial Agents 45(2): 130-36

Pulit-Penaloza, J. A.., Esser, E. S., Vassilieva, E. V., Lee, J. W., Taherbhai, M. T., Pollack, B. P., Prausnitz, M. R., Compans, R. W., and Skountzou, I. 2014. A Protective Role of Murine Langerin+ Cells in Immune Responses to Cutaneous Vaccination with Microneedle Patches. Scientific Reports 4: 1-9.

Sanchez, G. V., Master, R. N., Clark, R. B., Fyyaz, M., Duvvuri, P., Ekta, G., and Bordon, J. 2013. Klebsiella pneumoniae Antimicrobial Drug Resistance, United States, 1998-2010. Emerging Infectious Diseases 19(1): 133-36.

Sekowska, A., Gospodarek, E., and Kusza, K. 2014. The Prevalence of Infections and Colonisation with Klebsiella pneumoniae Strains Isolated in ICU Patients. Anaesthesiology Intensive Therapy 46(4): 280-83.

Sharma, A., Steichen A. L., Jondle, C. N., Mishra, 
B. B., and Sharma, J.. 2014. Protective Role of Mincle in Bacterial Pneumonia by Regulation of Neutrophil Mediated Phagocytosis and Extracellular Trap Formation. Journal of Infectious Diseases 209(11): 1837-46.

Shon, A.S., Bajwa, R. P. S., and Russo, T. A. 2013. Hypervirulent (Hypermucoviscous) Klebsiella pneumoniae: A New and Dangerous Breed. Virulence 4(2): 107-18.

Soares, A. C., Souza, D. G., Pinho, V., Vieira, A. T., Barsante, M. M., Nicoli, J. R., and Teixeira M. 2003. Impaired Host Defense to Klebsiella pneumoniae Infection in Mice Treated with the PDE4 Inhibitor Rolipram. British Journal of Pharmacology 140(5): 855-62.

Tsao, N., Kuo, C.F., Chiu, C. C., Lin, W. C., Huang, W. H., and Chen, L. Y. 2015. Protection against Klebsiella pneumoniae Using Lithium Chloride in an Intragastric Infection Model. Antimicrobial Agents and Chemotherapy 59(3): 1525-33.

Tzouvelekis, L. S., Markogiannakis, A., Psichogiou, M., Tassios, P.T., and Daikos G.L. 2012. Carbapenemases in Klebsiella pneumoniae and Other Enterobacteriaceae: An Evolving Crisis of Global Dimensions. Clinical Microbiology Reviews 25(4): 682707.

Villa, A., Rodriguez-Orozco, J. A. R., Lozano, C. B., and Rojo, C. C. 2012. The Increased Expression of CD11c and CD103 Molecules in the Neutrophils of the Peripheral Blood Treated with a Formula of Bacterial Ribosomes and Proteoglycans of Klebsiella pneumoniae. Arch Bronconeumol 48(9): 316-19.

Viscardi, R. M., Kaplan, J., Lovchik, J. C., He, J. R., Hester, L., Rao, S., and Hasday, J. D. 2002. Characterization of a Murine Model of Ureaplasma urealyticum Pneumonia.
Infection and Immunity 70(10): 5721-29.

Whitfield, C., Perry, M. B., MacLean, L.L., and Yu, S.H. 1992. Structural Analysis of the O-Antigen Side Chain Polysaccharides in the Lipopolysaccharides of Klebsiella Serotypes O2(2a), O2(2a,2b), and $\mathrm{O} 2(2 \mathrm{a}, 2 \mathrm{c})$. Journal of Bacteriology 174(15): 4913-19.

Woods, D. R., Peyton, J.C., Franklin, G. A., Turina, M., and Cheadle, W.G. 2007. Decreased Neutrophil Response in a Model of Chronic Intraperitoneal Klebsiella Infection. Surgical Infections. 8(3): 367-76

Wu, C.C., Lin, C. T., Cheng, W.Y., Huang, C. J., Wang, Z. C., and Peng, H. L. 2012. FurDependent MrkHI Regulation of Type 3 Fimbriae in Klebsiella pneumoniae CG43. Microbiology (Reading, England) 158(4): 1045-56.

Wutzler, P., Hardt, R., Knuf, M., and Wahle, K. 2013. Targeted Vaccine Selection in Influenza Vaccination. Deutsches Ärzteblatt International. 110:793-98.

Xiong, H., Carter, R. A., Leiner, I. M., Tang, Y. W., Chen, L., Kreiswirth, B.N., and Pamer, E. G. 2015. Distinct Contributions of Neutrophils and CCR2+ Monocytes to Pulmonary Clearance of Different Klebsiella pneumoniae Strains. Infection and Immunity 83(9): 3418-27.

Yang, C.S., Tsai, H. Y., Sung, C. S., Lin, K. H., Lee, F. L., and Hsu, W. M. 2007. Endogenous Klebsiella Endophthalmitis Associated with Pyogenic Liver Abscess. Ophthalmology 114(5): 876-80.

Yoo, D. G., Floyd, M., Winn, M., Moskowitz, S.M., and Rada, B. 2014. NET Formation Induced by Pseudomonas Aeruginosa Cystic Fibrosis Isolates Measured as Release of Myeloperoxidase-DNA and Neutrophil Elastase-DNA Complexes. Immunology Letters. 160(2): 186-94.

\section{How to cite this article:}

Ítalo Sousa Pereira, Maria Poliana Leite Galantine, Igor Pereira Ribeiro Muniz, Diego Patrick Soares Lopes, Karine Bitencourt Rodrigues, Lorena Santos Leal, Palloma Porto Almeida, Larissa Rodrigues de Oliveira Sousa and Robson Amaro Augusto da Silva. 2017. Intradermal Immunization of BALB/C Mice with Heat Killed Klebsiella pneumoniae ATCC BAA1706 Leads to the Production of both IgG1 and IgG2a. Int.J.Curr.Microbiol.App.Sci. 6(12): 2525-2537.

doi: https://doi.org/10.20546/ijcmas.2017.612.293 